\title{
»Du sollst dir kein Bildnis machen«
}

\author{
Religiöse Themen bei Max Frisch
}

Matthias Zeindler

\section{Ein religiöser, ein christlicher, ein theologischer Schriftsteller?}

Ist Max Frisch ein religöser, ein christlicher, ist er gar ein theologischer Schriftsteller? Einige neuere Publikationen können einen zu diesem Eindruck verleiten. Nur drei Beispiele:Peter von Matt schreibt in einem Essay über Frisch und Dürrenmatt, die Unterschiede zwischen den beiden Grossen der Schweizer Literatur hätten auch »ihre theologischen Aspekte«; bei Frisch sei es seine »Selbsterlösungslehre« ${ }^{1}$ Beatrice von Matt wird in ihrem schönen Buch »Mein Name ist Max Frisch" ausführlicher und ortet in Frischs Werk verschiedene Orte religiöser Erfahrung, so die Liebe, aber - überraschender vielleicht - auch das Meer, die Weite und Grenzenlosigkeit des Meeres. Sie weist nach, dass Max Frisch in gewissen Passagen das Meer unmissverständlich zu seinem Gott erklärt. ${ }^{2}$ Und ein dritter bekannter Literaturkritiker, Andreas Isenschmid, berichtet in der NZZ am Sonntag ausführlich über den »theologische[n] Urantrieb « in Frischs Schreiben, und er scheut nicht davor zurück, von einem Abschnitt in Stiller zu behaupten, er gehöre "als Beispiel einer Theologie ohne Gott in jedes theologische Lesebuch « ${ }^{3}$

Als Theologe freut einen so viel religiöse und theologische Sensibilität unter zeitgenössischen Literaturkritikern und -kritikerinnen. Selber würde man sich so etwas kaum trauen, aus Angst davor, der christlichen Vereinnahmung einer grossen Figur der Schweizer Literatur verdächtigt zu werden. Es gibt aber noch andere Gründe, die mich zögern lassen, Max Frisch zu schnell zum Gewährsmann für religiöse und christliche Gehalte zu machen. Da ist zunächst

1 P. von Matt, Sprengmeister, in: Der kleine Bund, 10. Oktober 2009, 9.

2 B. von Matt, Mein Name ist Max Frisch. Begegnungen mit dem Autor und seinem Werk, München 2011, 44.

3 A. Isenschmid, Seine Meisterschaft erlangte er erst nach sechzig, NZZ am Sonntag, 30. Januar 2011, Beilage, 14. 
sein Begräbnis. Die Totenfeier für Max Frisch fand auf seinen eigenen Wunsch zwar in einer Zürcher Kirche, in St. Peter, statt, aber ohne Pfarrer, ohne Segen, ohne Amen. Hugo Loetscher berichtet allerdings, dass Frisch die Feier zunächst im noch repräsentativeren Grossmünster geplant habe, wo man sie aber abgelehnt habe - Zwinglis Kirche sei "keine Mehrzweckhalle«. ${ }^{4}$ So oder so: eine Kirche. Ein religiöser Kontext, aber keine religiöse Sprache und keine christlichen Zeichen. In diesem Arrangement steckt eine ordentliche Ambivalenz, sicher aber muss man es zunächst als deutliches Zeichen der Abgrenzung ernstnehmen.

Ich bin der Meinung, dass sich diese Ambivalenz bei deutlicher Abgrenzung auch im Spätwerk von Max Frisch des öftern wiederholt. In diesem Spätwerk tritt das Religiöse weitgehend zurück. Weitgehend, aber eben nicht ganz. Nehmen wir das Stück Triptychon von 1978. Thema ist der Tod, der Tod als Abschluss von Lebensmöglichkeiten und als Verendgültigung des Gelebten. Eine religiöse Thematisierung des Todes sucht man in diesem Stück vergeblich. Ausser vermutlich an einer Stelle - präzis in der Mitte des Stücks und an Ostern, erklingt Glockengeläut und ein gregorianisches Te Deum. Genau an der Stelle, wo der Tod auch in seiner religiösen Dimension thematisch werden müsste, nimmt Frisch die Sprache zurück und überlässt einem anderen Medium das Wort. Es wäre zuviel gesagt, hier von einer religiösen Aussage zum Tod zu sprechen. Aber es wäre ebenso zuviel gesagt, würde man behaupten, Frisch schliesse eine religiöse Aussage zum Tod aus.

Weitere Beispiele, als erstes die Erzählung Montauk von 1975. Frisch erzählt von Tagen mit seiner Freundin Lynn, er hält Rückblick auf sein Leben und setzt sich mit dem Älterwerden auseinander. Wieder fehlt Religiöses. Ausser an einer Stelle, wo Lynn ihn fragt: »Do you believe? « (glaubst du) - die Frage bleibt unbeantwortet. In der grossartigen Erzählung Der Mensch erscheint im Holozän (1979) wird der geistige Zerfall eines alternden Mannes in einem durch Unwetter von der Umwelt abgeschnittenen Tal geschildert. Ein Satz lautet: "Ob es Gott gibt, wenn es einmal kein menschliches Hirn mehr gibt, das sich eine Schöpfung ohne Schöpfer nicht denken kann, fragt sich Herr Geiser. $\|^{5}$ Mehr als die Frage wird nicht notiert. Und im dritten Tagebuch, erst letztes Jahr erschienen, zum literarischen Werk seines Freundes, des sterbenden Juristen Peter Noll: »Meine

\footnotetext{
4 H. Loetscher, Lesen statt Klettern. Aufsätze zur literarischen Schweiz, Zürich 2003, $251 \mathrm{f}$.

5 Der Mensch erscheint im Holozän, Frankfurt a.M. 1979, 17.
} 
Angst vor Religiosität. $\ll^{6}$ Das Thema taucht auf, der Autor bleibt aber höchst zurückhaltend.

Immer wieder stösst man beim späten Frisch aber auch auf überraschende Stellen. Ich möchte hier zwei Fragen aus Fragebögen im Tagebuch 1966-1971 erwähnen. Im Fragebogen zum Thema Humor lesen wir: "Gesetzt den Fall, Sie glauben an Gott: kennen Sie ein Anzeichen dafür, dass er Humor hat? ${ }^{7}$ Und im Fragebogen zum Thema Eigentum: "Was gefallt Ihnen am Neuen Testament? ${ }^{8}$ - eine Frage, die im Zusammenhang gerade mit dem Eigentum an Hintergründigkeit kaum zu überbieten ist.

Alles in allem lässt sich vom späten Frisch kaum sagen, er sei ein religiöser, ein christlicher oder ein theologischer Schriftsteller. Am angemessensten versteht man ihn als Agnostiker, in dessen Horizont religiöse Fragen vorkommen, ohne dass er sich dazu positiv äussern könnte. Wenn er sich äussert, dann am ehesten im Modus der Frage oder der bewussten Leerstelle.

Anders stellt sich die Sache dar im frühen und mittleren Werk von Max Frisch. Hier ist es angebracht, von eigentlichen religiösen Themen zu sprechen, welche in den betreffenden Texten eine bestimmende Rolle spielen. Ich möchte auf drei solche Themen etwas ausfuhhrlicher eingehen. Ich tue dies nicht als Germanist, sondern als Theologe. Also zum einen als literaturwissenschaftlicher Dilettant, zum anderen mit einem speziellen Augenmerk gerade auf die religiösen Dimensionen in den besagten Texten. Beginnen wir mit einem ausgesprochenen Frühwerk.

\section{Antwort aus der Stille}

Max Frischs zweite Buchpublikation erscheint 1937 unter dem Titel Antwort aus der Stille. Eine Erzählung aus den Bergen. In späteren Jahren scheint der AutorVorbehalte gegen dieses kleine Buch entwickelt zu haben, er hat es jedenfalls nicht in die in den 70er Jahren erscheinende Ausgabe seiner Gesammelten Werke aufgenommen. Erst 2009 ist es wieder neu aufgelegt worden. ${ }^{9}$

Die erzählte Geschichte ist einfach, fast parabelhaft: Ein junger Mann entschliesst sich in einer Lebenskrise zu einer lebensgefährlichen Erstdurchsteigung eines Bergrates. Im Zusammenhang mit die-

6 Entwürfe zu einem dritten Tagebuch, Frankfurt a.M. 2010, 114

7 GW 6, 203

8 A.a.O., 377.

9 Frankfurt a.M. 2009. Seitenzahlen im Text. 
ser Tour kommt es auch zu einer kurzen, heftigen Liebesbeziehung zu einer Frau. Bei seinem Versuch entgeht der Mann nur ganz knapp dem Tod und kehrt ohne seinen rechten Arm zurück. Innerlich aber hat er sich gewandelt, er hat seine Krise überwunden und eine neue Perspektive auf sein Leben gewonnen.

Zentral geht es in dem Buch um zwei Einstellungen zum Leben. Ausführlich wird in den Reflexionen und Gesprächen vor der entscheidenden Bergtour die Haltung geschildert, welche den jungen Mann - erst ganz gegen Ende erfährt man seinen Namen: Balz - in die Krise geführt hat. Sein bisheriges Leben beurteilt er so:

"Da erkennt man keinen klaren Ablauf und keinen roten Faden, da zerrinnt es einfach, ohne Abschnitt und ohne Tat, die Leidenschaft zerrinnt in eine Stimmung, und auch die Entschlüsse sind wie Sand, der leise durch die Finger rinnt, immer wieder nimmt man eine neue Handvoll, und wenn man sie aufmacht, ist wieder nichts darin geblieben, man ist verzweifelt; und auch das zerrinnt, wie die Hoffnung und der Jubel und der Schmerz und alles, wie das ganze Leben." (47)

Man fühlt sich an den Prediger Salomo erinnert - alles ist eitel und ein Haschen nach Wind. Es ist die Frage nach dem wirklichen, dem wahren Leben, welche den jungen Frisch hier umtreibt - und übrigens noch durch Jahrzehnte umtreiben wird. Er stellt fest, wie leer doch ist, was wir gewöhnlich als Leben bezeichnen.

\footnotetext{
"Und was nichts anderes sei, sagt er, als ein Kette von verlorenen Tagen, die immer hinter ihren Plänen zurückbleiben; Wochen und Jahre vergehen, und man verstünde es nicht, oft meine man, es sei alles nur ein einziger Tag, ein grosser Alltag, der immer der gleiche ist: wenn man sich auszieht und die Zähne putzt, wie gestern und schon seit Jahren, und wenn man noch eine Weile auf dem Bettrand sitzt und wieder den Wecker aufdreht, langsam und mit der unleugbaren Einsicht, dass man wieder nichts erfüllt hat, wie gestern und schon seit Jahren.«(81)
}

Und sein Fazit lautet:

"Nein! sagt er nur und schüttelt den Kopf: das sei es ja eben, dann wolle er schon lieber nicht leben, so nicht, als gewöhnlicher Mensch nie und nimmer!« (83)

Es ist das bürgerliche, das alltägliche, das verzettelte und fragmentierte Leben, welches Frischs Protagonist verabscheut, das für ihn den Tod schon im Leben bedeutet. Und der Weg auf den Berg, die 
gefährliche Herausforderung ist natürlich der Gegenentwurf dazu, die Realisierung des nicht gewöhnlichen, des intensiven, des erfüllten Lebens. So fragt der junge Bergsteiger seine Geliebte einmal unvermittelt:

"Ob sie denn mitkommen würde? Einfach mitkommen, auf und davon, über alle Berge? [...] In irgendein Land, sagt er, wo es keinen Alltag gebe, wo man keinen Menschen kenne, wo man wirklich leben könnte, ohne Bindung und ohne Rücksicht, ohne alles, was nicht dazu gehört, ein wirkliches Leben, ein Leben ohne Gewöhnung, ein Leben voll Erlebnis, ein Leben, wie es unsere Sehnsucht kennt, ein neues und anderes, ein lebenswertes Leben -!« (96)

Die Suche nach dem wahren Leben scheitert, endet haarscharf vor dem Tod. Und wird darin doch gerade zur Wende zu einem wahren Leben. Freilich zu einer anderen Wahrheit, als sie der junge Mann intendiert hat. Denn als wahres Leben erschliesst sich ihm mit einem Male gerade das verachtete, das gewöhnliche Leben. Dasjenige, das er doch hinter sich lassen wollte. Nicht der waghalsige Weg auf den Gipfel führt ihn am Ende ins Leben. Sondern der Abstieg ins Tal. Das Bild dieses Weges ins Tal gerät Frisch zum berauschenden Hymnus:

"Ein neuer und klarer Morgen wird aufgehen, ein gläsernes Leuchten, eine silberne und blitzende Glut, wenn die Sonne wieder über die stummen Berge steigt und über die Täler, wo es grüne Matten gibt und Wälder, die nach Harz und Schwämmen duften, und immer tiefer wird man steigen, an den ersten Hütten vorbei, wo die schwarzen Ziegen bimmeln und wo auch wieder Blumen sind, die im Morgentau glitzern, und wieder wird es ein blauer und nicht allzu warmer Tag sein, ein zarter und goldener Tag, als ob nichts geschehen sei, ein stilles und friedliches Tal, wo man durch Dörfer kommt und wieder Menschen sieht, die dich grüssen, wo die Frauen am hölzernen Brunnentrog waschen, wo alles tätig ist, und an den Hängen draussen werden die Männer stehen und den Roggen schneiden, der inzwischen reif geworden ist, mit schwingenden und leise singenden Sensen -« (145)

Und so ist der letzte Satz der Erzählung ein grosses Ja:

"Dass es ein unsagbar ernstes Glück ist, leben zu dürfen, und dass wohl nirgends die Leere sein kann, wo dies Gefuihl auch nur einmal wirklich errungen worden ist, dies Gefühl der Gnade und des Dankes." (Ebd.) 
»... der Gnade und des Dankes«: das sind theologisch hochgradig gefüllteWörter.Wir kommen damit zu den theologischen Dimensionen der Antwort aus der Stille. ${ }^{10}$ Der gesamte Gang dieser Erzählung wird begleitet durch einen Bogen von ausdrücklich religiösen Aussagen, und ich bin der Meinung, man kann die Bedeutung dieser Aussagen nicht überschätzen. So erlebt der Bergsteiger die Gebirgswelt als "Gottes steile und silberne Handschrift» (35), als "namenlose Stille, die vielleicht Gott oder das Nichts ist « (36). Die Berge sind für ihn nicht eindeutig als Fingerabdruck Gottes lesbar, wie das unsere moderne Naturromantik so gerne hat. Indem Gott sich in ihnen äussert, entzieht er sich auch, wird er fremd und fraglich. Auch sonst begegnet Gott nicht als gütig-väterlicher Gott. Der Mensch muss etwas tun, heisst es später, nicht "einfach dasitzen und ins Leere schauen und nachdenken, was Gott, in seiner unergründlichen Langeweile, vielleicht mit dem Menschen gemeint hat" (42f.). Ein anderes Bild für das Leben sieht so aus: Das Leben ein Zirkus, alles rennt im Kreis, in der Mitte "der liebe Gott, der uns mit hundert Leidenschaften peitscht, damit sein Spass nicht ausstirbt, und der lach $[t]$ sich den Bauch voll« (50). Und gegen Ende des Buches reisst die Bergwelt nochmals eine erschreckende Sicht auf Gott auf, in einer einsamen Bergnacht, in der es ist, "als zeige Gott ein anderes Antlitz [...], ein wahres Antlitz vielleicht, das nichts von Menschen weiss und ohne Erbarmen scheint, ohne Gnade gegen das Leben, so stumm und starr, so steinern und fremd« (128).

Wie gesagt, kein gütig-väterlicher Gott wird in diesen Sätzen gezeichnet. Ein fremder Gott vielmehr, ein kalter, selbstgenügsamer Gott, der sich ob der Welt langweilt, sie gar verhöhnt, auf den wir uns jedenfalls nicht verlassen und an dem wir uns nicht orientieren können. Diese Vorstellung von Gott hat aber im Erzählganzen ihre Konsequenz. Denn im Bild des kalten, gnadenlosen Gottes spiegelt sich sehr genau die Kälte des Lebensentwurfes des Protagonisten: der einsame Zwang, ein aussergewöhnliches Leben um jeden Preis aus eigener Kraft zu realisieren. Das religiöse Erleben des jungen Mannes entspricht seiner Selbsterfahrung, seine Theologie seiner Anthropologie. Wenn dies richtig ist, dann müsste sich seine Wandlung auch in einer Wandlung seines Gottesbildes zeigen. Das ist tatsächlich der Fall, wenn auch in sehr verhaltender Weise, und zwar in dem bereits zitierten Schlusssatz. Wo Balz das Leben vorher als Möglichkeit erfahren hat, die es unbedingt zu ergreifen gilt, so erschliesst es sich ihm nun als Gabe, als Geschenk. Und dem Geschenk begegnet man

\footnotetext{
${ }^{10}$ Eine Untersuchung des Wortes "Gnade« in Frischs Werk wäre ausserordentlich ergiebig.
} 
nicht mehr mit der vorher mehrmals erwähnten "männlichen Tat" man begegnet ihr mit Dank und im Bewusstsein, dass Leben Gnade ist.

Woher unser junger Mann dies übrigens weiss? Es kann sich bei dieser Erfahrung nur um die Antwort aus der Stille handeln, die im Titel genannt wird. $\mathrm{Ob}$ man Frisch theologisch überfrachtet, wenn man sie als das interpretiert, was in religiöser Terminologie Offenbarung heisst? Dabei muss man darauf achten, wo sich bei Frisch der Ort dieser Offenbarung befindet. Denn natürlich ist es die Bergwelt. Genau gesehen ist es aber das Scheitern, das in dieser Erzählung in der Bergwelt stattfindet, aber auch anderswo stattfinden könnte. Im Scheitern seines Lebensplans wird dem Bergsteiger die Wahrheit offenbar. Drei Nächte war er übrigens verschollen. $\mathrm{Ob}$ man aus dieser Zeitangabe einen Hinweis auf die Auferstehung herauslesen kann, lasse ich als Frage stehen.

\section{3. ॥Du sollst dir kein Bildnis machen ...»}

Wir kommen zu einem weiteren religiösen, ja, zu einem christlichen Thema in Max Frischs Werk: zu seiner spezifischen Interpretation des Bilderverbots. Es gibt kaum jemanden, dem diese Thematik bei Frisch nicht geläufig ist. Ich möchte es deshalb an diesem Punkt bei einigen Bemerkungen bewenden lassen.

Im Tagebuch 1946-1949 findet sich ein Text, den Pfarrerinnen und Pfarrer sehr gerne bei Hochzeiten lesen:

"Es ist bemerkenswert, dass wir gerade von dem Menschen, den wir lieben, am mindesten aussagen können, wie er sei.Wir lieben ihn einfach. Eben darin besteht ja die Liebe, das Wunderbare an der Liebe, dass sie uns in der Schwebe des Lebendigen hält, in der Bereitschaft, einem Menschen zu folgen in allen seinen möglichen Entfaltungen. [...] Die Liebe befreit aus jeglichem Bildnis. Das ist das Erregende, das Abenteuerliche, das eigentlich Spannende, dass wir mit den Menschen, die wir lieben, nicht fertigwerden: weil wir sie lieben; solange wir sie lieben. «11

Wenige Seiten nach dieser Passage beginnt eine Prosaskizze unter dem Titel Der andorranische Jude, und an deren Ende finden wir folgende Sätze:

${ }^{11}$ GW II, 369. 
"Du sollst Dir kein Bildnis machen, heisst es, von Gott. Es dürfte auch in diesem Sinne gelten: Gott als das Lebendige in jedem Menschen, das, was nicht erfassbar ist. Es ist eine Versündigung, die wir, so wie sie an uns begangen wird, fast ohne Unterlass wieder begehen -

Ausgenommen wenn wir lieben. $«^{12}$

Der andorranische Jude ist eine von zahlreichen Skizzen später ausgearbeiteter Werke in den ersten von Frischs Tagebüchern; diese Skizze fand ihre endgültige Gestalt bekanntlich später im Theaterstück Andorra, einer Parabel, in welcher ein junger Mann, Andri, von einer Dorfbevölkerung für einen Juden gehalten wird, immer eindeutiger für einen Juden, bis er selbst bei sich alle möglichen Merkmale eines Juden feststellt. Nachdem Andri umgebracht worden ist, stellt sich heraus, dass er kein Jude war, sondern ein uneheliches Kind seines Vaters, des Lehrers. Zwischen den Szenen treten die Personen des Stücks einzeln gleichsam in den Gerichtsstand und äussern sich im Rückblick. Der Pater bezieht in seinem Auftritt das Problem ausdrücklich auf das Bilderverbot. Er kniet dabei:
»Du sollst dir kein Bildnis machen von Gott, deinem Herrn, und nicht von den Menschen, die seine Geschöpfe sind.Auch ich bin schuldig geworden damals. Ich wollte ihm mit Liebe begegnen, als ich gesprochen habe mit ihm. Auch ich habe mir ein Bildnis gemacht von ihm, auch ich habe ihn gefesselt, auch ich habe ihn an den Pfahl gebracht. $1^{13}$

Wir begegnen dem Zusammenhang von Bilderverbot und Liebe in Frischs Werk an zahlreichen Stellen. Fragen kann man nun, ob es sich bei ihm dabei wirklich noch um eine religiöse Thematik handelt. Unbestritten, Frisch bezieht sich auf einen klassisch religiösen Bestand aus der biblischen Tradition, auf eines der zehn Gebote. Gleichzeitig gibt er an, dass er dieses Gebot ausdrücklich aus einem religiösen in einen zwischenmenschlichen Kontext transferiert "Gott als das Lebendige in jedem Menschen«. Verliert das Gebot damit seinen theologischen Gehalt, geht es in seiner zwischenmenschlichen Geltung auf? Ist, wenn Frisch vom Göttlichen spricht, dieses mithin bloss noch metaphorisch zu verstehen?

Ich würde hier vor einer zu eindeutigen Antwort warnen. Zum einen habe ich nicht den Eindruck, dass sich die Frage an Frischs Werk eindeutig entscheiden lässt. Zum andern gibt es aber auch gute

\footnotetext{
12 A.a.O., 374.

13 GW IV, 509.
} 
theologische Gründe, sie offenzulassen. Denn natürlich spricht das zweite Gebot im Alten Testament ausdrücklich davon, dass man sich von Gott kein Kultbild anfertigen soll. Und natürlich findet sich eine Übertragung des Bilderverbots auf den Menschen in der Bibel nicht. Wohl aber wird der Mensch im ersten Kapitel der Bibel als Bild Gottes bezeichnet: »Und Gott sprach: Lasst uns Menschen machen nach unserem Bild, uns ähnlich" (Gen 1,26). Diese Gottebenbildlichkeit des Menschen macht ihn zwar nicht göttlich, sie begründet aber seine unbedingte, unantastbare Würde als Geschöpf. Und kraft dieser Würde ist der Mensch berechtigt, nicht eingeschlossen zu werden in das Korsett eines Bildes, das wir uns voneinander machen. Es ist Teil seiner Freiheit, mehr zu sein als die Bilder von ihm. Es ist diese theologische Dimension menschlicher Würde, welche Max Frisch mit seinem Bezug auf das Bilderverbot festhält. Sie wäre anders als so nicht ausdrückbar und ist deshalb in der Tat ein religiöses Thema in seinem Werk.

\section{Auferstehung im Roman »Stiller«}

An letzter Stelle möchte ich auf Max Frischs grossen Roman Stiller eingehen. Ich halte dieses Buch für einen, wenn nicht den Höhepunkt in Frischs Schaffen. Stiller ist nicht zuletzt im Blick auf die Auseinandersetzung mit religiösen Fragen ein Höhepunkt im Werk von Frisch.

Ganz kurz zum Inhalt: Der Roman hat einen der berühmtesten ersten Sätze: "Ich bin nicht Stiller." Damit ist das Programm des Buches angeben - der Ich-Erzähler ist bei der Einreise in die Schweiz gefangen genommen und wegen eines Spionageverdachts eingekerkert worden. Die Behörden gehen davon aus, dass er der seit sechs Jahren verschollene Bildhauer Anatol Stiller ist. Was der Erzähler konsequent bestreitet, obwohl man es ihm mit immer eindeutigeren Beweisen nachweist. Am Ende wird er vom Gericht dazu verurteilt, Stiller zu sein. Gleichgewichtig neben dieser Identitätsproblematik steht der Bericht über Stillers Ehe, wobei die beiden Themen eng verbunden sind. Das Ganze wird von einerVielzahl von anderen Geschichten und Reflexionen unterbrochen, die aber alle die Thematik variieren, spiegeln oder erläutern; dazu gehört nicht zuletzt eine beissende Kritik an der real exisierenden Schweiz. Damit ist Stiller ein ungemein reicher, komplexer und vor allem auch sprachlich grosser Roman, der ohne Zweifel zu den wichtigsten der Literatur im 20. Jahrhundert gehört. 
Von Religiösem handeln dabei verschiedene Stellen, unter anderem bringt Stillers Frau Julika ebenfalls einmal den Bezug zum Bilderverbot ins Spiel. Auch liest Stiller in seiner Gefängniszelle regelmässig die Bibel. Aber Gott taucht bei ihm stets nur in der Form des Zweifelns und des Fragens auf. In konzentrierter Form geht es um die Gottesfrage im zweiten, kurzen zweiten Teil des Romans, dem Nachwort des Staatsanwalts, der zu diesem Zeitpunkt schon während längerer Zeit auch Stillers Freund ist. Rolf erweist sich in diesem Teil des Buches als religiöser Mensch, als derjenige, der Gott immer wieder in direkter Weise anspricht. Dass Rolf diesen Gott Stiller "katechetisch anzudemonstrieren sucht", wie der Theologe Christoph Gellner behauptet, halte ich für dem Text völlig unangemessen. ${ }^{14}$ Der Staatsanwalt vertritt im Romanganzen eine ernstzunehmende Stimme, welcher gegenüber weder der Autor noch Stiller selbst eindeutig Stellung nehmen. Rolf spricht weder für Max Frisch noch für Anatol Stiller, er spricht aber für eine Position, die ins Geflecht der unterschiedlichen Stimmen dieses Buches unveräusserlich hineingehört.

Der Schlussteil dreht sich auf der einen Seite nochmals betont um die Frage, wie ein Mensch sich als der annehmen kann, der er nun mal ist. Das war ja der grosse Konflikt, den Stiller im Gefängnis mit den Behörden und mit seiner Umwelt ausgefochten hatte. Dieser Kampf ist zum Zeitpunkt des Nachworts abgeschlossen. Stiller ist nun frei, er lebt mit seiner Frau in einem kleinen Haus am Genfersee. Es ist ihm, so sein Staatsanwalt, gelungen, sich selbst anzunehmen. Offen bleibt für den Juristen und Freund die Frage, auf welche Weise man mit seiner Selbstannahme leben kann. Für ihn ist klar: "Es wird nie möglich sein ohne die Gewissheit, dass unser Leben von einer übermenschlichen Instanz gerichtet wird, ohne wenigstens die leidenschaftliche Hoffnung, dass es diese Instanz gebe." Er fügt hinzu: »Stiller kam spät dazu. Kam er dazu? «15 Was will der Staatsanwalt mit dem Satz von der notwendigen äusseren Instanz sagen? Er spricht hier eine tiefe Einsicht aus, die insbesondere in der Reformation starkgemacht worden ist. Die Einsicht nämlich, dass wir als Menschen das Ja zu uns selbst nicht selber verbürgen können. Unser Ja zu uns bleibt immer unter der Frage, ob es denn berechtigt sei, ob es der Wirklichkeit entspreche. Allein Gottes Ja zu uns setzt

\footnotetext{
14 "Sind wir schon dadurch, dass wir geboren worden sind, schuldig?» Biblisch-religiöse Urfragen im Werk des reformierten Bilderkritikers Max Frisch, in: J. Badewien /H. Schmidt-Bergmann (Hg.), »Jedes Wort ist falsch und wahr«. Max Frisch neu gelesen. Karlsruhe 2008, 112-138.123.

${ }^{15}$ GW III, 751. Seitenzahlen im folgenden im Text.
} 
diese Frage ausser Kraft, nimmt ihr jeden Zweifel. Andernfalls gleichen wir dem Baron Münchhausen, der sich - und sein Pferd - am eigenen Zopf aus dem Sumpf gezogen hat. Unser Ja zu uns trägt erst, wenn es von Gottes Ja getragen wird.

Stillers Probleme sind mit seiner Selbstannahme freilich noch nicht gelöst. Darum geht es in der zweiten Hälfte des Schlussabschnittes. Denn nicht nur sich selbst hatte er bisher nicht angenommen, auch seine Frau hatte er bislang nicht als den Menschen gelten lassen können, der sie wirklich war. Die Dramatik dieser Verfehlung treibt die letzten Seiten des Romans an. Der Staatsanwalt Rolf und seine Frau sind zum zweiten Mal bei Stiller zu Besuch. Unvermittelt und zu Stillers grösster Bestürzung - rückt Frau Julika Stiller mit lebensbedrohlichem Lungenkrebs ins Spital ein. Zur Deutung der religiösen Dimension dieser Passagen ist es entscheidend wichtig, dass die letzten Tage und Stunden des Romans am Osterwochenende stattfinden. Darauf wird mehrmals hingewiesen. Ostern: Das Fest der Auferweckung Christi. In diesem Horizont wollen die abschliessenden Gespräche zwischen Stiller und seinem Freund gelesen werden.

In der Nacht von Ostern auf Ostermontag, früh um eins, trifft Rolf in dessen Wohnzimmer auf den schon stark angetrunkenen Stiller. Stiller ist in einem elenden Zustand. Plötzlich bricht es aus ihm heraus: »Rolf - ich kann einen Menschen töten, aber ich kann ihn nicht wieder auferwecken ...» (762) Sie kann von mir nichts mehr annehmen, sagt er. "Lass mich«, hört er von ihr. Und fragt dann:»Warum habe ich diese Frau nie gefunden? Nie! Nicht einen Tag lang, Rolf, nicht eine Stunde lang in dieser ganzen Zeit. Nie!« (763) Rolf fragt Stiller zurück, was er denn von seiner Frau und sich erwartet habe. $\mathrm{Ob}$ er eine Verwandlung erwartet habe. Und hält dann dagegen:

"Manchmal könnte man fast meinen, du hältst dich für einen Zauberer, der diese Frau Julika in ihr Gegenteil verzaubern kann. [...] Auferwecken! Dein alter lieber Unsinn, Stiller, du gestattest, dass ich es dir sage: dein mörderischer Hochmut - Du als Erlöser eurer selbst!« (764)

Hier kommt sie, die Auferweckung, der österliche Akt. Aber nicht als Tat Gottes, sondern als angemasste Tat eines Menschen.

Rolf verdeutlicht nochmals, was er meint. Er hält Stiller vor, dass er seine Wichtigkeit für seine Frau überschätze. Stiller stutzt, und er antwortet ihm:

"Ja, diese Frau hat dich nie zu ihrer Lebensaufgabe gemacht.

Nur du hast so etwas aus ihr gemacht, glaube ich, von allem 
Anfang an. Du als ihr Erlöser, ich sagte es schon, du wolltest es sein, der ihr das Leben gibt und die Freude. Du! In diesem Sinn hast du sie geliebt, gewiss, bis zum eignen Verbluten. Sie als dein Geschöpf.« (765)

Hier liegt die Hybris von Stiller, in diesem Wunsch, durch seine Liebe seine Frau zu verändern. Der Autor ihres Glücks zu sein und damit ihr eigentlicher Schöpfer. Vergessen wir nicht Stillers Beruf, Bildhauer. Das ist sein Verständnis von Liebe: einen Menschen nach seinem Willen zu formen. Mit diesem Wunsch verfehlt er seine Frau, sich selbst und die Liebe. Stiller möchte mit seiner Liebe seine Frau "auferwecken", sie neu machen. Gerade dieses Bedingungsverhältnis muss aber aufgelöst werden, soll die Liebe zwischen diesen beiden Menschen eine Chance haben.

Stiller bohrt in immer neuen Anläufen, wie Rolf sich denn das Zusammenleben zwischen Julika und ihm unter diesen neuen Bedingungen vorstelle. Darauf Rolf:

„Es gibt keine Änderung, ihr lebt miteinander, du mit deiner Arbeit da unten im Souterrain, sie mit ihrer halben Lunge, so Gott will, und der einzige Unterschied: ihr foltert euch nicht mehr Tag für Tag mit dieser irren Erwartung, dass wir einen Menschen verwandeln können, einen anderen und uns selbst, mit dieser hochmütigen Hoffnungslosigkeit [...] Ganz praktisch: Ihr lernt beten für einander.« (772)

Diesen letzten Satz kann man ganz präzis theologisch verstehen. Es geht in ihm keineswegs einfach um Erbaulichkeit. Zu beten füreinander, das ist der eigentliche Gegenentwurf zum Versuch, den anderen Menschen gemäss den eigenen Vorstellungen umzubauen. Indem wir für einen Menschen beten, geben wir ihn aus unserer eigenen Hand. Wir geben ihn frei. Und wir geben ihn derart frei, dass wir ihn in die Hand eines anderen, eines Grösseren geben. Aus dem Unser Vater wissen wir, all unser Beten muss unter dem Vorbehalt »Dein Wille geschehe« erfolgen. Indem wir für einen andern Menschen beten, verzichten wir darauf, unseren eigenen Willen zum Massstab dieses Menschen zu machen. Im Gebet geben wir einen Menschen frei - darum ist das Gebet ein tiefer Ausdruck für die Liebe. Stiller wehrt sich nicht gegen dieses Votum seines Freundes. Er fügt lediglich an: »Beten will gekonnt sein!« (ebd.) Daraufhin schweigt er.

Am Tag darauf begibt sich Rolf ins Spital, wo er vom Tod Julikas erfährt. An ihrem Totenbett erfasst er nochmals die tiefe Lebensproblematik seines Freundes: 
"plötzlich hatte ich das ungeheure Gefühl, Stiller hätte sie von Anfang an nur als Tote gesehen, zum ersten Mal auch das tiefe, unbedingte, von keinem menschlichen Wort zu tilgende Bewusstsein seiner Versündigung.« (779)

Nochmals: Versündigung geschieht da, wo wir annehmen, ein Mensch komme erst durch uns zum Leben, sei ohne uns tot. Wir müssten ihn - und sei es durch unser Edelstes, unsere Liebe - verändern, zu einem wahreren Menschen machen. Wo wir einen Menschen aber annehmen, sehen wir ihn als einen, der ohne uns, vor unserem Tun, schon lebt. Der sein Leben also von anderswo her als von uns bekommen hat. Hier wird nun der österliche Hintergrund des Romanendes sprechend: Auferweckung zum Leben steht uns nicht zu, sie wird uns gewährt. Auferstehung, Zum-Leben-Kommen geschieht, wo wir dies erfassen. Und wo wir es für uns und die Menschen neben uns gelten lassen.

Wir haben es hier, bei Max Frisch, mit einem Versuch zu tun, die Auferstehungsthematik im Bezug auf das Diesseits zu reformulieren. Und für Frisch heisst das insbesondere: sie im Bezug auf die menschlichen Beziehungen zu reformulieren. Wir kennen diesen Gedanken schon von seiner Umformulierung des Bilderverbots her. Wie für Frisch das 2. Gebot erfüllt wird durch die Liebe, die sich vom Nächsten kein Bildnis macht, so erfüllt sich für ihn auch die Auferstehung in der Liebe, die einen Menschen annimmt, wie er ist. Wobei man auch hier nicht wird sagen können, Frisch habe dadurch die Auferstehung säkularisiert und betreibe so eine streng horizontale Hermeneutik. Vielmehr ist sein Roman darin ganz klar, dass menschliche Liebe es nicht ist, welche das Annehmen meiner selbst und des andern bewerkstelligt. Nein, menschliche Liebe - und das ist nun ein durch und durch theologischer Gedanke -, menschliche Liebe ist nichts anderes als die tätige Anerkennung der Tatsache, dass der andere und ich selbst von Gott schon angenommen sind. Eine Interpretation von »Stiller», welche diese Dimension nicht einbezieht, vermag m.M. den Roman nicht in seiner Tiefe auszuschöpfen.

\section{Schluss}

Zum Schluss nochmals die Frage, ob Max Frisch ein religiöser, ein christlicher Schriftsteller sei. Die erste Antwort muss ohne Zweifel lauten: Nein. Nicht nur wegen seiner eingestandenen Angst vor dem Religiösen, sondern vor allem deshalb, weil in seinem Werk andere Themen zentral sind. 
Aber - und das ist die zweite Antwort - einige seiner Themen behandelt Frisch in seinem Werk auch unter einer religiösen Perspektive. Ich habe das am Werk bis zu Stiller zu zeigen versucht. Und es ist für eine Deutung dieses Werks nicht gut, wenn man diese Dimension vernachlässigt. ${ }^{16}$

Nach Stiller verstärkt sich bei Frisch die Skepsis. Die Leerstellen in seinen Texten werden grösser, allgemein und besonders in Sachen Religion. Die Fragen bleiben, die Antworten werden aber leiser und am Ende ganz zurückgehalten. So kann man vielleicht auch das Begräbnis von Max Frisch deuten: als eine Frage ohne Antwort. Nicht zuletzt darin besteht bis heute die Modernität Max Frischs.

\footnotetext{
- Dr. Matthias Zeindler ist Titularprofessor für systematische Theologie an der Theologischen Fakultät der Universität Bern.

${ }^{16}$ J. Bauke-Ruegg vertritt die Auffassung, dass die Literaturwissenschaft "je länger je mehr auf die Tätigkeit der Theologie angewiesen« ist. Sein Argument: Literatur - und besonders auch Gegenwartsliteratur - hat in vielen Fällen eine religiöse bzw. theologische Dimension, die zu entschlüsseln in einer zunehmend religiös analphabetischen Gesellschaft immer weniger Lesende in der Lage sind. Auch bei Literaturwissenschafterinnen und -wissenschaftern kann eine entsprechende Kenntnis nicht mehr selbstverständlich vorausgesetzt werden. »Insofern die Auslegung literarischer Texte als eigentliche Aufgabe der literaturwissenschaftlichen Arbeit angesehen wird, ist die kritisch-rationale Arbeit der Theologie, die expliziert, dass literarische Texte theologische Fragestellungen, Motive,Vorstellungen und Formen aufgreifen und wie sie sie aufgreifen, von unmittelbarer literaturwissenschaftlicher Relevanz (Theologische Poetik und literarische Theologie? Systematisch-theologische Streifzüge, Zürich 2004, 593f.). Die hier vorgelegte Interpretation von Stiller bestätigt Baukes These. In negativer Weise wird sie bestätigt durch die Tatsache, dass besonders die auferstehungstheologischen Aspekte in Frischs Roman bisher von der Literaturwissenschaft nicht wahrgenommen wurden - obwohl sie nur schwer zu übersehen sind.
} 